\title{
New approach to genome-wide automated inference of bacterial transcription factor binding sites
}

\author{
Yevgeny Nikolaichik \\ Department of Molecular Biology \\ Belarusian State University \\ Minsk, Belarus \\ nikolaichik@bsu.by
}

\begin{abstract}
We describe a method for identification of transcription factor binding sites (TFBS) in bacterial genome sequences. The method is based on two bits of information: (i) specific contacts between amino acid residues of DNA-binding domains and DNA nitrogen bases deduced from 3D structures of DNA-protein complexes and (ii) transcriptional units' organisation inferred from genome annotation. The method can be useful for correct application of experimentally verified TFBS motifs to new genomes and also for discovering TFBS motifs de novo. Method was verified with known Escherichia coli TFBSs and evaluated during genome-wide TFBS inference in Pectobacterium spp. which showed its high efficiency.
\end{abstract}

Keywords - transcription factor binding sites; operator; regulon; genome annotation; motif discovery

\section{Motivation and aim}

Motivation

Current approaches to bacterial genome annotation are mostly restricted to coding sequences, rRNA, tRNA and in some cases ncRNA genes. None of the popular pipelines can identify regulatory elements (transcription factor binding sites, promoters and terminators), which makes functional annotation incomplete and reduces the usability of genome sequences.

Aim

To develop a reliable method, based on experimental data, for automated inference of transcription factor binding sites in bacterial genomes.

\section{Methods}

Transcription factors (TFs) were classified according to models of DNA-binding domains present in PFAM [1]. Protein and DNA motif searches were performed with hmmsearch and nhmmer from the HMMER package [2]. Transcription factor binding site (TFBS, also called operator) motif identification and comparison were done with programs from the MEME suite package [3]. Clustering of the regulatory regions to reduce redundancy was done with MeShClust [4]. Transcriptional terminators were identified with TransTermHP [5]. Pipeline glue code, regulatory region analysis, operon inference logic and graphical interface were written in Xojo and integrated into a previously developed SigmoID program [6].

Genome sequences of Escerichia coli K-12 substr. MG1655 (accession code NC 000913), Pectobacterium atrosepticum 21A (CP009125) and $P$. versatile 3-2 (CP024842) were used for testing TFBS inference algorithms.

\section{Results}

Sahota and Stormo have suggested using the information on 3D structures of TF-operator complexes for de novo

\author{
Pavel Vychik \\ Department of Molecular Biology \\ Belarusian State University \\ Minsk, Belarus \\ p.vychik@gmail.com
}

inference of TFBS motifs [7]. The idea is based on (i) conservation of positions of amino acid residues within DNA binding domain (critical residues) that directly contact DNA bases and (ii) regulation by most TFs of their own or neighbouring genes.

We extend this idea by attaching critical residue information (CR-tag) to experimentally verified TFBS profiles. As CR tag specifies the amino acid residues making specific contacts with DNA bases within an operator, TFs with the same CR-tag can recognise the same operators. This approach allows to correctly apply experimentally determined TFBS profiles available from such databases as RegulonDB and CollecTF to genomes with no experimental TFBS data. If a genome being analysed encodes a TF with a CR-tag identical to the one in an experimentally characterised TF than a TFBS profile for the characterised TF can be safely applied to finding TFBSs in the target genome.

The success of the CR-tag based approach described above depends on the representativeness of the motif library. Its current version contains over 2000 profiles and is based on RegulonDB and CollecTF data supplemented with a selection of profiles from RegPrecise and few profiles collected from the literature. This library appears to have profiles with CRtags matching about half of TFs of an average enterobacterium. CR-tag matches are less frequent outside Enterobacteriales and this frequency drops to just 1-2 \% of total $\mathrm{TF}$ number for Gram-positive bacteria. However, a modified de novo approach to TFBS motif identification described below could be applied in such cases.

We have significantly improved the original de novo TFBS motif inference algorithm of Sahota and Stormo (Fig. 1). Slow protein database searches were replaced with fast CR-tag lookup tables, regulatory region extraction was improved (first $50 \mathrm{bp}$ of ORFs and divergons are now taken into the account), redundancy elimination was improved by two clustering stages (TFs and their regulatory regions are both clustered and representatives of each cluster proceed to the next stage), motif finding with meme is run in two modes (any number and zero or one per sequence). Finally, a comparison step was added to aid in choosing the correct motif among several ones usually found. The last comparison step of the pipeline is especially important since it provides solid criteria for choosing the correct motif out of several possible ones. When testing the pipeline with known TFs, we have usually found that correct TFBS motif not only matched one or few other motifs for TFs from the same family with sufficiently low p-value, but these TFs also had similar CRtags. Running the improved pipeline with E. coli genome demonstrated that it finds known motifs for over $90 \%$ of TFs that respect method assumptions. 


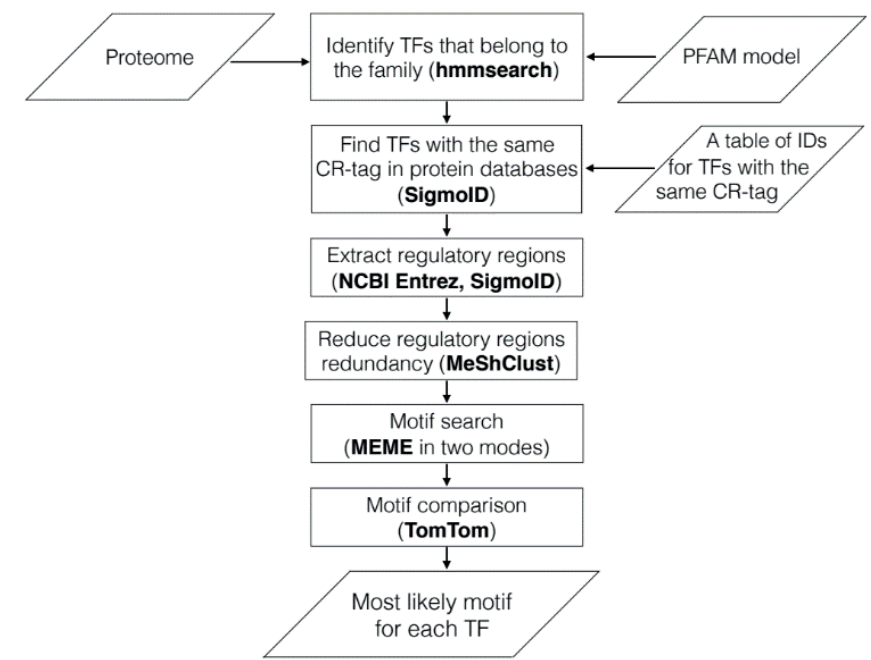

Fig. 1. De novo TFBS inference pipeline

Complications with the generally quite efficient de novo TFBS inference protocol may arise due to several factors: the absence of 3D structures of TF-operator complexes, presence of two DNA-binding domains in a TF (as in most TFs from the AraC family), indels within CR-tag region, absence of autoregulation (quite common in the LuxR family), the formation of heterodimers with other TFs and so on. To estimate the efficiency of the de novo protocol with a nonmodel organism, we have performed a full scan of Pectobacterium atrosepticum genome. Out of $258 \mathrm{TFs}$ found by hmmscan with PFAM models, 164 TFs could be used for CR-tag based de novo TFBS motif inference procedure. Based on CR-tag matches to known motifs, motif comparison and correspondence to motif features, typical for TF families (like T-N11-A pattern for LysR family, direct repeats for LysR family and so on) and few P. atrosepticum motifs characterised experimentally, correct motifs were found for 124 (76\% of the analysed, $48 \%$ of total) TFs.

We also tried to evaluate the predictive power of TFBS inference while looking for new regulatory circuits during genome-wide TFBS analysis. We scanned P. atrosepticum and $P$. versatile genomes with the de novo inferred TFBS profiles to find regulons controlled by each TF. Since pectobacteria are plant pathogens, special attention was paid to TFs that change their expression levels in planta according to available RNA-seq data $[8,9]$. Few interesting observations from this analysis are listed below. LacI family TF binding site analysis combined with expression levels changes reported by RNA-seq allowed to functionally link multiple beta-glucoside utilisation loci scattered across the genome; LuxR and OmpR family analysis revealed unexpected new details of quorum sensing regulation, LysR family analysis found new global regulator of secondary metabolism, etc. We are currently verifying these in silico predictions experimentally.

\section{Conclusions}

The method of TFBS identification described here is universal (can be applied to any genome sequence of any bacterium) and can be expected to find up to half of TFBSs present in a genome with minimal user intervention. We expect method efficiency to improve if pipeline parameters are fine-tuned for a particular genome and TF family and with a further extension of CR-tag based motif library. Our genome-wide TFBS inference approach is capable of discovering unknown regulatory circuits. We advocate its wide use for generating testable hypotheses for further experimental research of bacterial regulatory networks. Source code and executables of the user-friendly GUI application implementing the method described here are available from github.com/nikolaichik/SigmoID.

\section{ACKNOWLEDGMENT}

This work was supported by a grant from Belarusian Republican Foundation for Basic Research and the State program "Biotechnology".

\section{REFERENCES}

[1] R. D. Finn et al., "The Pfam protein families database: towards a more sustainable future," Nucleic Acids Res, vol. 44, no. D1, pp. D279D285, Jan. 2016, doi: 10.1093/nar/gkv1344.

[2] S. R. Eddy, “Accelerated Profile HMM Searches," PLoS Comput Biol, vol. 7, no. 10, p. e1002195, Oct. 2011, doi: 10.1371/journal.pcbi.1002195.

[3] T. L. Bailey, J. Johnson, C. E. Grant, and W. S. Noble, "The MEME Suite," Nucl. Acids Res., vol. 43, no. W1, pp. W39-W49, Jan. 2015, doi: 10.1093/nar/gkv416.

[4] B. T. James, B. B. Luczak, and H. Z. Girgis, "MeShClust: an intelligent tool for clustering DNA sequences," Nucleic Acids Res, vol. 46, no. 14, pp. e83-e83, Aug. 2018, doi: 10.1093/nar/gky315.

[5] C. Kingsford, K. Ayanbule, and S. Salzberg, "Rapid, accurate, computational discovery of Rho-independent transcription terminators illuminates their relationship to DNA uptake," Genome biology, vol. 8, no. 2, p. R22, 2007.

[6] Y. Nikolaichik and A. U. Damienikan, "SigmoID: a user-friendly tool for improving bacterial genome annotation through analysis of transcription control signals," PeerJ, vol. 4, p. e2056, May 2016, doi: 10.7717/peerj.2056.

[7] G. Sahota and G. D. Stormo, "Novel sequence-based method for identifying transcription factor binding sites in prokaryotic genomes," Bioinformatics, vol. 26, no. 21, pp. 2672-2677, Nov. 2010, doi: 10.1093/bioinformatics/btq501.

[8] V. Gorshkov et al., "Transcriptome profiling helps to identify potential and true molecular switches of stealth to brute force behavior in Pectobacterium atrosepticum during systemic colonization of tobacco plants," European Journal of Plant Pathology, May 2018, doi: 10.1007/s10658-018-1496-6.

[9] D. Bellieny-Rabelo, C. K. Tanui, N. Miguel, S. Kwenda, D. Y. Shyntum, and L. N. Moleleki, "Transcriptome and Comparative Genomics Analyses Reveal New Functional Insights on Key Determinants of Pathogenesis and Interbacterial Competition in Pectobacterium and Dickeya spp.," Appl. Environ. Microbiol., vol. 85, no. 2, pp. e02050-18, Jan. 2019, doi: 10.1128/AEM.02050-18. 